\title{
Absorptionsuntersuchungen an der sichtbaren Bande des Joddampfes
}

\author{
Von Werner LucK \\ Aus dem Institut für Physikalische Chemie an der Universität Tübingen \\ (Z. Naturforschg. 6 a, 313-319 [1951]; eingegangen am 23. April 1951)
}

\begin{abstract}
Es werden die scheinbaren Extinktionskoeffizienten des Joddampfes bei $5461 \AA$ bestimmt. Schon bei konstanter Schichtdicke sind hier bei verschiedenen Drucken positive und negative Abweichungen vom Lambertschen Gesetz zu beobachten. Durch Zusatz von Stickstoff wird erreicht, daß das Lambertsche Gesetz gültig wird, was durch den Übergang der Rotationsstruktur des Spektrums in ein Kontinuum gedeutet werden kann. Unsere Messungen sind innerhalb der experimentellen Fehlergrenze durch die Berechnungen der Gesamtabsorption überlappender Spektrallinien quantitativ zu erklären.
\end{abstract}

$\mathrm{I}_{\mathrm{d}}^{\mathrm{n}}$ n der vorangehenden Arbeit ${ }^{1}$ konnten wir zeigen, daß die kürzlich durchgeführten Auswertungen der von $\mathrm{E} \mathrm{l}$ s a s s e r formulierten Gesamtabsorption überlappender Spektrallinien² für die Deutung der Bromdampfabsorption herangezogen werden können. Diese Auswertungen erweisen sich als noch besser geeignet zur Deutung unserer Joddampfmessungen.

\section{Experimentelles}

Es wurde die Gesamtabsorption des Joddampfes'mit der in II beschriebenen lichtelektrischen Substitutionsmethode im Bereich der grünen $\mathrm{Hg}$-Linie bestimmt. Wegen des geringeren Joddampfdruckes mußten die Messungen aber bei $210^{\circ} \mathrm{C}$ durchgeführt werden, dafür konnte folgende experimentelle Verbesserung eingeführt werden: Die Jodmengen wurden auch hier mit einer Mikrowaage in Schmelzpunktsröhrchen eingewogen. An die Vorratsgläschen wurde jedoch vorher ein Schliff angesetzt, so daß die Gläschen evakuiert und dann abgeschmolzen werden konnten. Wir benutzten reines Jod mit Garantieschein der Firma M e r c k, das in einem Exsiccator über gereinigter (wie in II beschrieben) $\mathrm{H}_{2} \mathrm{SO}_{4}$ aufbewahrt wurde. Die Reproduzierbarkeit der Jodmessungen war etwas besser als die der entsprechenden Brommessungen. Die gleiche Erfahrung haben schon Rabinowits ch und Wood ${ }^{3}$ bei ihren Rekombinationsversuchen gemacht. Es wird vermutet, daß Spuren von $\mathrm{H}_{2} \mathrm{O}$ bei Brom stärker stören ${ }^{4}$. Eine weitere Vergrößerung der Fehlergrenze ist möglicherweise bei Brommessungen in einer Änderung des Reflexionsvermögens an den Küvettenabschlußplatten zu suchen. Bei unserer Methodik zur Bestimmung des Lambertschen Gesetzes (L.G.) $I=I_{0} e^{-\varepsilon N z}$ wird $I_{0}$ bei leerer Küvette gemessen und $I$ bei gefüllter. Das gemessene $I_{0}{ }^{\prime}$ entspricht aber nicht dem Wert von $I_{0}$, welcher nach dem

1 G. K o r t ü m u. W. L u c k, Z. Naturforschg. 6a, 305 [1951], im folgenden als II zitiert.

2 W. L u c k, Z. Naturforschg. 6a, 191 [1951], im folgenden als I zitiert.

3 E. Rabinowitch u. W. C. Wood, Trans. Farad. Soc. 32, 912 [1936].
Durchlaufen der Schicht $z$ erreicht wird, sondern durch das Reflexionsvermögen $(R)$ der Küvettenabschlußplatte messen wir $I_{0}{ }^{\prime}=I_{0}-R I_{0}=I_{0}(1-R)$. Bei gefüllter Küvette hat zwar die reflektierte Energie den anderen $\mathrm{Be}$ trag: $R I_{0} e^{-\varepsilon N z}$, gemessen wird aber

$$
I=I_{0} e^{-\varepsilon N z}-R I_{0} e^{-\varepsilon N z}=I_{0}{ }^{\prime} e^{-\varepsilon N^{\top} z} .
$$

Bei konstantem Reflexionsvermögen bleibt also der reflektierte Bruchteil der Lichtintensität gleich, und das L.G. wird dadurch nicht beeinflußt. Die Veränderung der Reflexion durch die Änderung des Brechungsindexes in der Küvette nach dem Einlassen der Halogendämpfe ist innerhalb der Fehlergrenze. Wohl aber wäre es möglich, daß sich an der Quarzwand auf Grund der chemischen Aggresivität des Broms eine Bromadsorptionsschicht bildet, die den Brechungsindex in Wandnähe meßbar verändert. Weniger glauben wir, daß sich bei unseren Untersuchungen die Änderung des Absorptionsvermögens innerhalb der adsorbierten Schichten bemerkbar macht. Die von $\mathrm{Golup}$ und $\mathrm{K}$ ondrat jew ${ }^{5}$ nachgewiesene Absorptionserhöhung durch adsorbiertes $\mathrm{NO}_{2}$ konnte auch in der gleichen Anordnung bei Joddampf von $\mathrm{Har}$ d in g ${ }^{6}$ nicht gefunden werden.

\section{Meßergebnis se}

Abb. 1 zeigt die so in einem Druckbereich von 0,8 bis $105 \mathrm{~mm}$ (umgerechnet auf $0^{\circ} \mathrm{C}$ ) gemessenen scheinbaren Extinktionskoeffizienten des reinen Joddampfes. Dort ist $\bar{\varepsilon}$ in $l / \mathrm{Mol} \mathrm{cm}$ (Skala auf der linken Abbildungsseite) als Funktion von $\mathrm{Nz}$ in $\mathrm{Mol} \mathrm{cm} / \mathrm{l}$ (untere Skala) aufgetragen. Als Parameter ist die Schichtdicke einzusetzen. Sie steigt von oben nach

4 K. F. L uf t (Z. Physik 84, 767 [1933]) hat bei Messungen der Dielektrizitätskonstanten des Bromdampfes auch die Schwierigkeit festgestellt, die letzten Spuren von $\mathrm{H}_{2} \mathrm{O}$ zu beseitigen.

5 S. G o l u p u. V. K o n d rat j e w, Physik. Z. Sowjetunion 1, 619 [1931].

6 J. H a r d i n g, Philos. Mag., J. Sci. 21, 773 [1936]. 


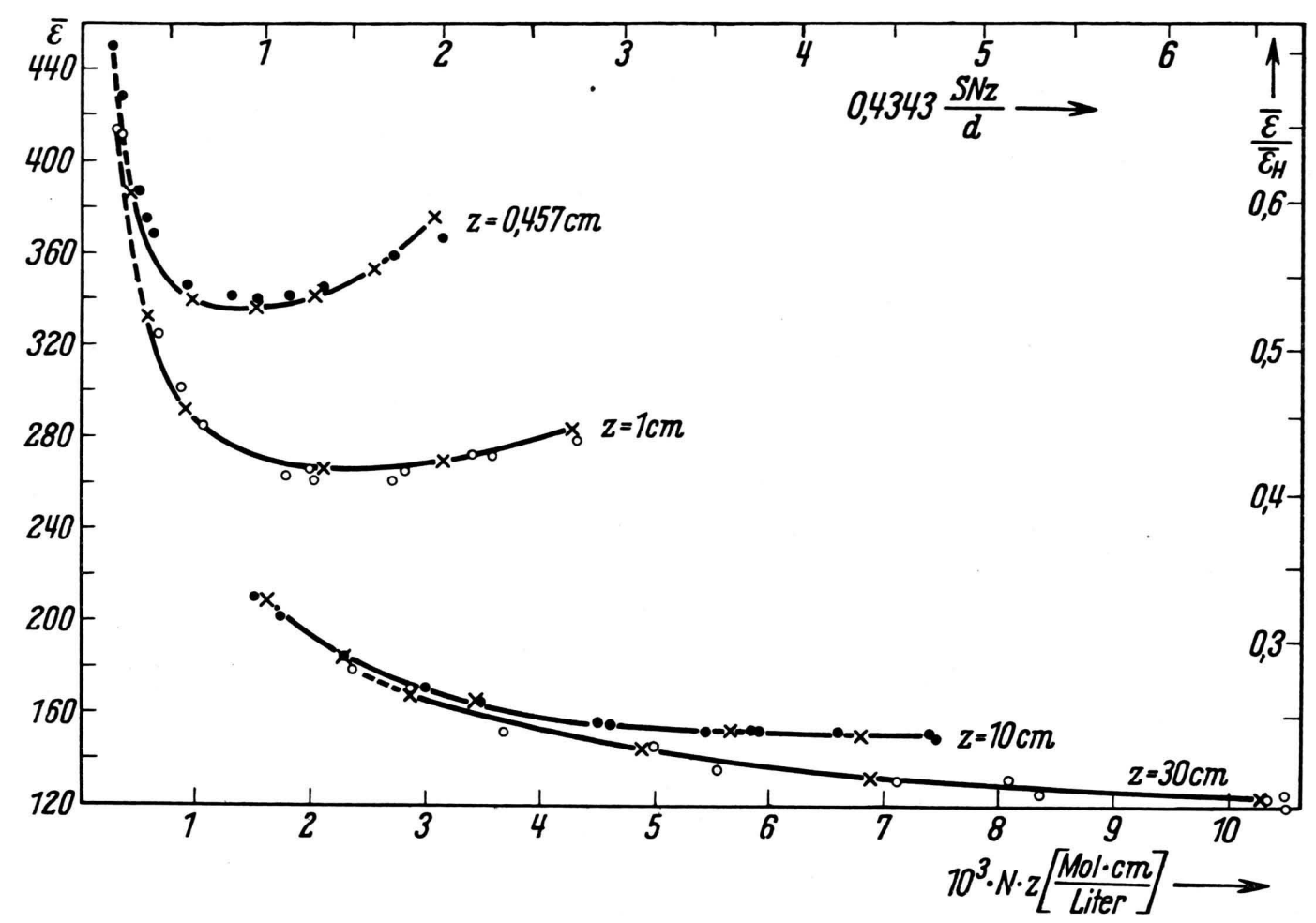

Abb. 1. Scheinbare Extinktionskoeffizienten von Joddampf. $\backslash$ theoretisch; $\bigcirc$ und $\bullet$ experimentell.

unten von $0,475 \mathrm{~cm}$ bis $30 \mathrm{~cm}$. Beim Jod kommen demnach schon bei gleicher Schichtdicke sowohl mit dem Druck fallende als auch steigende Extinktionskoeffizienten vor. Dazwischen im Gebiet des Minimums wird also das L.G. annähernd gelten. Bei kleinen $\mathrm{Nz}$-Werten sind die Unterschiede zwischen den Kurven verschiedener Schichtdicken geringer, das Beersche Gesetz (B.G.) wird dort eher erfüllt sein.

In Abb. 2 sind die gemessenen Extinktionen für $z=1 \mathrm{~cm}$ als Funktion der Molekülzahl $N$ aufgetragen. Diese Abbildung zeigt die Anforderungen an die

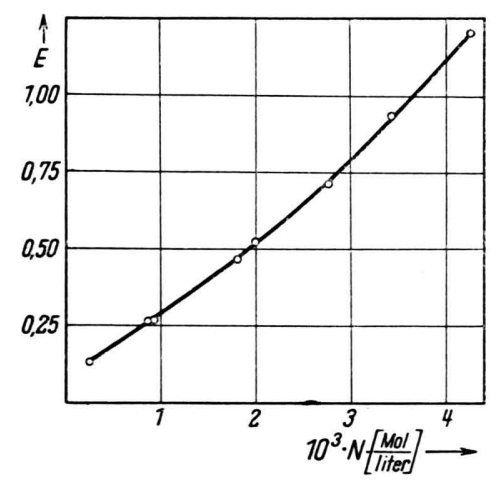

Abb. 2. Extinktion von Joddampf.
Genauigkeit bei der Umrechnung auf Abb. 1. Ferner ist zu ersehen, daß bei der häufigen Nachprüfung der Absorptionsgesetze in der Form der Abb. 2 wesentliche Feinheiten verlorengehen können. Selbst in dem Fall starker Änderungen von $\bar{\varepsilon}$ mit $N$ sind die Abweichungen von einer Geraden in Abb. 2 verhältnismäßig gering.

Auch hier können wir die bisher bekannt gewordenen Jodmessungen in unserem größeren Meßbereich einordnen. D lug o s $\mathrm{ch}^{7}$ fand das B.G. bis zu Joddrucken von $1 \mathrm{~mm}$ bestätigt. Seine Messungen dürften in Abb. 1 wohl in dem engzusammenlaufenden Gebiet der $\mathrm{zu} z=10 \mathrm{~cm}$ und $30 \mathrm{~cm}$ gehörenden Kurven liegen.

Auch die Messungen von $\mathrm{Koehler}^{8}$ mit $z=10 \mathrm{~cm}$ und $4 \mathrm{~cm}$ bei Extinktionen bis 0,07 sind im Bereich des linken Endes unserer Kurven durchgeführt worden. Nach K o e hl e r gilt hier das B.G. Aber selbst aus seiner groben Darstellungsart (Absorptionsprozente gegen $N z$ ) ist zu erkennen, daß die Meßpunkte für $4 \mathrm{~cm}$ einer höheren Absorption als für $10 \mathrm{~cm}$ Schichtdicke entsprechen.

7 F. G. D l u g o s c h, Diss. Breslau 1923.

8 J. F. K o e h l e r, Physic. Rev. 44, 761 [1933]. 
H a rding ${ }^{6}$ beobachtete bei $5461 \AA$, daß die Absorption direkt dem Druck proportional sei. $z$ wird bei ihm etwa $2 \mathrm{~cm}$ betragen haben. Auch $\mathrm{H}$ a r d in $\mathrm{g}$ stellte fest, daß die für niedrigere Drucke extrapolierte Gerade wie in unserer Abb. 2 nicht in den Nullpunkt mündet. Aus dieser Abweichung muß, wie schon in I erwähnt, auf eine Ungültigkeit des L.G. geschlossen werden. Hardings Ergebnisse entsprechen dem annähernd geradlinigen Kurventeil in Abb. 2.

Starke Absorptionserhöhungen mit dem Druck fanden dagegen $\mathrm{Kondratjew}$ und $\mathrm{Polak}{ }^{9}$, wenn sie den Druck von 0,2 bis $107 \mathrm{~mm}$ mit $z=0,455$ bis $200 \mathrm{~cm}$ variierten. Dieser Anstieg entspricht auch etwa dem von uns erfaßten Druckbereich.

$\mathrm{N}$ a k a m u r a ${ }^{10}$ macht lediglich die qualitative Angabe, daß der Joddruck die gleiche absorptionserhöhende Wirkung wie die Druckerhöhung bei Fremdgaszusatz hat.

Für die Auswertung unserer Messungen nach dem in I angegebenen Rechenverfahren ist die Kenntnis der Sättigungs-Extinktionskoeffizienten bei durch hohen Druck verschmierter Rotationsstruktur erforderlich. Da dieses Druckgebiet bei hohem Jodeigendruck wegen der großen Extinktionskoeffizienten schwieriger zu erfassen ist, haben wir es durch Zusatz von Stickstoff durchgemessen, der in diesem Spektralbereich nicht absorbiert.

Zur Untersuchung von Jod-Stickstoff-Mischungen wurden wieder 4 Jodmengen in der beschriebenen Weise bereitgestellt (vgl. II.), die 1-cm-Küvette evakuiert, dann mit reinem Stickstoff gefüllt und abgeschmolzen. Es wurde nachgereinigter Stickstoff der Firma L i n d e benutzt, er wurde über $\mathrm{P}_{2} \mathrm{O}_{5}$ getrocknet. Der Druck wurde mit einem Hg-Manometer beim Einfüllen bestimmt.

Den Abb. 3 und 4 sind die Ergebnisse unserer Messungen mit $\mathrm{J}_{2}-\mathrm{N}_{2}$-Mischungen $\mathrm{zu}$ entnehmen. Wegen der langsamen Diffusionsgeschwindigkeit dauerten die Messungen mit vier verschiedenen Jodmengen hier bis zu 18 Stdn., so daß eine höhere Fehlergrenze in Kauf genommen werden mußte. Das gilt vor allem für die höheren Stickstoffdrucke. Bei diesen Messungen mußte angenommen werden, daß die Nullkompensation der Photozellen bis zu 4 Stdn. erhalten blieb. In der Abb. 3 sind die scheinbaren Extinktionskoeffizienten in gleicher Art wie in Abb. 1 aufgetragen. Zum Vergleich mit Abb. 1 ist die dortige Kurve für reines Jod bei $z=0,475 \mathrm{~cm}$ auch in Abb. 3 eingezeichnet worden. Für die übrigen

9 V.Kondrat je w u. L. P ol a k, Z. Physik Sowjetunion 4, 764 [1933].

10 G. N a k a mu ra, Mem. Coll. Kyoto Imp. Univ., Ser. A, 9, 315 [1925/26].

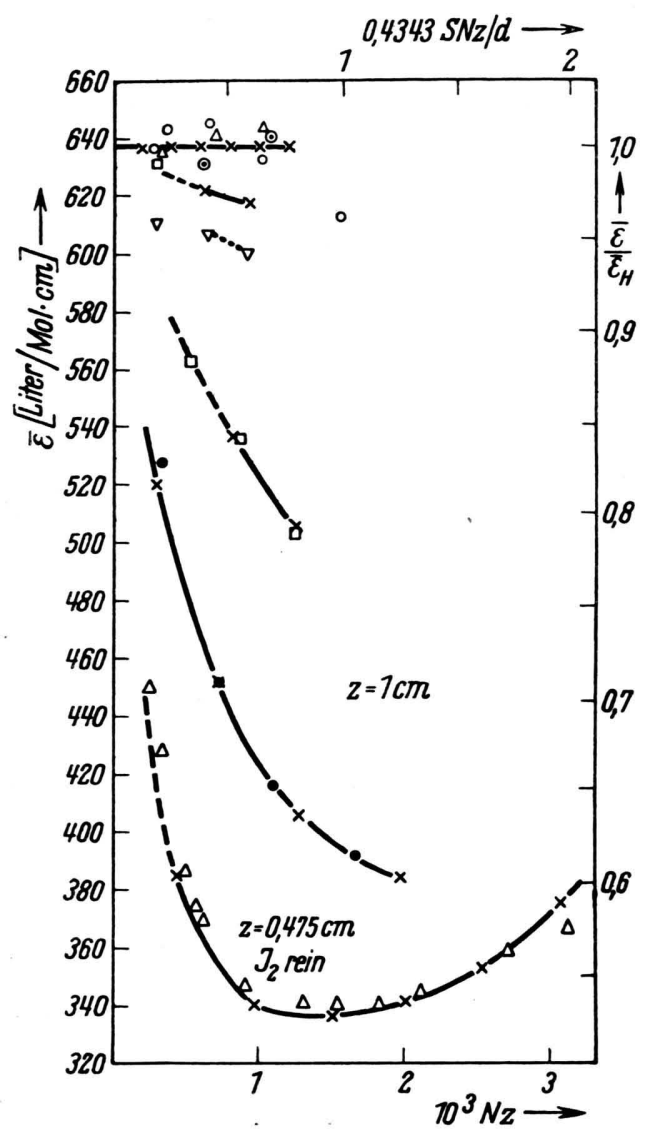

Abb. 3. $\mathrm{J}_{2}-\mathrm{N}_{2}$-Fremdgaseffekt. Theoretisch: $X$; experimentell: $\bullet 48 \mathrm{~mm}$; $\square 100 \mathrm{~mm}$; $\nabla 236 \mathrm{~mm}$; $309 \mathrm{~mm}$; $\triangle 450 \mathrm{~mm} ; \odot 730 \mathrm{~mm}$.

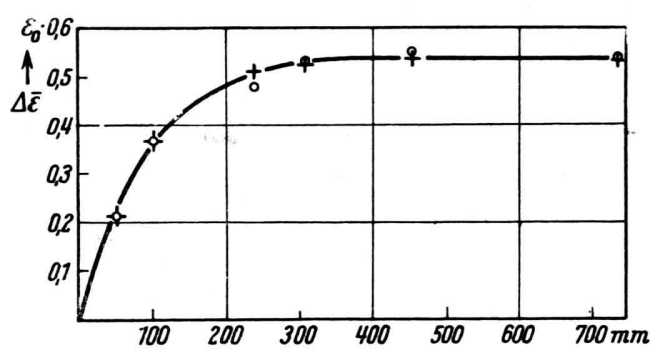

Abb. 4. $\mathrm{J}_{2}-\mathrm{N}_{2}$-Fremdgaseffekt als Funktion des $\mathrm{N}_{2}$-Druckes. $0,43 \frac{\mathrm{S} \mathrm{Nz}}{d}=0,6$.

Kurven ist als Parameter der Stickstoffdruck gewählt worden. Mit wachsendem Stickstoffdruck (die Druckangaben in Abb. 3 und 4 beziehen sich auf $20^{\circ} \mathrm{C}$ ) finden wir eine Zunahme von $\bar{\varepsilon}$, bis bei höheren $\mathrm{N}_{2}$-Drucken die $\bar{\varepsilon}$-Werte innerhalb der Fehlergrenze zusammenfallen. Abb. 4 stellt einen Schnitt durch 
Abb. 3 parallel zur Ordinatenachse beim Abszissenwert 0,6 der oberen Skala dar. Bis $300 \mathrm{~mm} \mathrm{~N}_{2}$-Zusatz haben schon $\mathrm{K}$ ondratjew und Polak $\mathrm{k}^{9}$ in dieser Weise Untersuchungen an $\mathrm{J}_{2}-\mathrm{N}_{2}$-Mischungen veröffentlicht, die mit unseren etwa übereinstimmen. Durch die Erweiterung unserer Messungen bis zu 1 at Fremdgaseffekt können wir jedoch die Existenz eines „Sättigungsgebietes“ sicher nachweisen. Mit dieser Erweiterung der Messungen kann auch gezeigt werden, daß der von diesen Verfassern ${ }^{9}$ vorgeschlagene Deutungsversuch nicht richtig sein kann. Für die Erhöhung des Extinktionskoeffizienten $\bar{\varepsilon}_{\bar{\varepsilon}}$ geben sie folgende Formel an:

$$
\lrcorner_{\bar{\varepsilon}}=C \frac{K p_{\mathrm{F}}}{1+K p_{\mathrm{F}}} .
$$

$C$ und $K$ sind Konstanten, $p_{\mathrm{F}}$ bedeutet den Fremdgasdruck. Später haben Avramenko und Kond r a t je w ${ }^{11}$ auch auf den Fremdgaseffekt des Bromdampfes Gl. (1) angewandt. Sowohl die Fremdgasmessungen an Bromdampf von $\mathrm{K}$ or $\mathrm{t} \ddot{\mathrm{u}} \mathrm{m}$ und $\mathrm{M}$ ü lle $\mathrm{r}$ als auch unsere an Joddampf lassen sich nicht durch (1) darstellen. Durch Umformung kann aus (1) erhalten werden:

$$
\frac{1}{K p_{\mathrm{F}}}=\frac{C}{\overline{\Delta \varepsilon}}-1 \text {. }
$$

$1 / \Delta \bar{\varepsilon}$ gegen $1 / p_{\mathrm{F}}$ aufgetragen müßte also nach (2) eine Gerade ergeben. Bei hohen Drucken wird aber nach den hiesigen Messungen $\bar{\varepsilon}$ im Widerspruch zu (2) konstant. Höchstens in dem von Kondratjew und P o l a k gemessenen Bereich bis $300 \mathrm{~mm}$ Druck kann (1) noch als Näherungsformel benutzt werden*. Das Versagen der Formel (1) liegt an dem Ansatz, daß dem Jodmolekül und einem $\mathrm{J}_{2}-\mathrm{N}_{2}$-Stoßpaar je ein Extinktionskoeffizient zugeordnet wird. Das ist hier nicht statthaft, da sich $\bar{\varepsilon}$ innerhalb des Auflösungsvermögens des benutzten Spektrographen auf Grund der Linienstruktur stark ändert, also eine Reihe verschiedener Extinktionskoeffizienten schon beim $\mathrm{J}_{2}$ allein gemessen werden.

11 L. Avramenko u. V. Kondratjew, Physik. Z. Sowjetunion 10, 741 [1936].

* Besser als durch Gl. (1) lassen sich die Bromfremdgasmessungen durch folgende empirische Näherungsformel darstellen:

$$
E=\ln \frac{I_{0}}{I}=p_{\mathrm{Br}_{2}} z\left[a+b e^{C p_{\mathrm{F}} /\left(p_{\mathrm{Br}_{2}}+p_{\mathrm{F}}\right)}\right] .
$$

Durch Reihenentwicklung der $e$-Funktion erhält man bei konstantem Bromdruck ( $\left.p_{\mathrm{Br} 2}\right)$ für kleine Fremddrucke einen Ausdruck von der Form (1) ( $a, b, c$ sind Konstante).

12 R. W. W o o d, Philos. Mag., J. Sci. 35, 230 [1918].

\section{Diskussion}

Unsere Untersuchungen an Joddampf lassen sich mit den in I entwickelten Formeln für die Gesamtabsorption überlappender Linien in befriedigender Weise quantitativ deuten. Wo od ${ }^{12}$ gibt die Zahl der Absorptionslinien der sichtbaren Joddampfbande mit 50000 an, so daß von den Voraussetzungen E ls a s s ers die Annahme unendlich vieler Linien hier annähernd gilt. Über die Annahme, daß die Linien alle die gleiche Linienbreite haben sollen, liegen keine Beobachtungen vor. Bei $\mathrm{HCN}$ ist zwar eine Abhängigkeit der Linienbreite von der Rotationsquantenzahl beobachtet worden ${ }^{18-17}$, Margenau und Wats o ${ }^{18}$ sowie $\mathrm{Margenau}$ und $\mathrm{W}$ arren ${ }^{19}$ haben aber gezeigt, daß diese Abhängigkeit nur bei Molekülen mit Dipolmomenten größer als $2 \cdot 10^{-18}$ c.g.s.Einheiten zu erwarten ist (vgl. auch ${ }^{19 a}$ ). Die Voraussetzung, daß die Jodlinien alle den gleichen Linienabstand haben, ist nach Feinstrukturuntersuchungen von $\mathrm{A} g$ a rbi c e a n u ${ }^{20}$ nicht gut erfüllt. Letzterer hat im Bereich der grünen Hg-Linien 4 Jodlinien ausgemessen, Opechowski ${ }^{21}$ hat dort 7 Jodlinien beobachtet. Angaben über das Intensitätsverhältnis der Jodlinien fehlen. Die Annahme gleich intensiver Linien ist hier aber wohl eher erfült als beim Bromspektrum, weil Jod keine Isotope besitzt. Die Idealisierungen, mit denen die Rechnungen in I durchgeführt wurden, sind demnach auch beim Jodspektrum nicht alle erfüllt, wir können aber versuchen, ob die in I entwickelten Ergebnisse von der Erfüllung der idealisierenden Annahmen empfindlich abhängen.

Im Einklang mit den Beobachtungen $\mathrm{Naka-}$ muras s ${ }^{10}$ und A garbice an u s ${ }^{20}$ nehmen wir an, daß das Sättigungsgebiet des Fremdgaseffektes durch sehr starke Linienverbreiterungen zu deuten ist. Wegen der höheren Fehlergrenze wurde der Grenzwert des scheinbaren Extinktionskoeffizienten $\bar{\varepsilon}_{\mathrm{H}}=0,4343 \mathrm{~S} / d$ durch Mittelbildung zu $637 \mathrm{l} / \mathrm{Mol} \mathrm{cm}$ aus unseren

13 G. Herzberg u. S. W. T. S p inks, Proc. Roy.

Soc. [London], Ser. A, 147, 434 [1934].

14 S. D. Cornell, Physic. Rev. 51, 595 [1937].

15 E. Li n d h o l m, Diss. Uppsala 1942.

16 H. N. F o le y, Physic. Rev. 69, 227 [1946].

17 G. Kortüm u. H. Verleger, Proc. physic. Scc. 63, 462 [1950].

${ }_{18} \mathrm{H}$. M a r g e n a u u. W. W. W a t s o n, Physic. Rev. 51, 48 [1937].

${ }_{19} \mathrm{H}$. M argen a u u. D. T. Warre n, Physic. Rev. 51, 748 [1937].

19a W. L a s a r ef f, Z. Physik 64, 598 [1930].

20 I. A g a r b i c e a n u, Ann. Physique 2, 397 [1934].

$\approx 1 \mathrm{~W}$. O p e chowski, Act. physic. polon. 3, 308 [1934]. 
Messungen bestimmt. Durch Division der Ordinatenwerte der Abb. 1 durch 637 und durch Multiplikation der Abszissenwerte mit 637 wurden die in der Abb. 1 eingezeichneten Skalen am oberen und rechten Rand erhalten. Durch Vergleich der so gedeuteten Abb. 1 mit der in I angegebenen Abb. 2 kann für jeden Meßpunkt das Verhältnis von Linienbreite zu Linienabstand $\beta$ bestimmt werden. Für die Auswertung der Messungen für $z=30 \mathrm{~cm}$ und $10 \mathrm{~cm}$ wurden die Erweiterungen der Abb. (I, 2) durch asymptotische Entwicklung der Bessel-Funktion nach Gl. $(I, 22)$ numerisch berechnet. Diese Auswertung ergab, daß $\beta$ bei jeder Schichtdicke linear mit dem Druck zunimmt. Die Minima in Abb. 1 kommen so zustande, daß beim Eintragen dieser Werte in das Rechenblatt (I, Abb. 2) mit wachsendem Druck die zu verschiedenen $\beta$-Werten gehörenden Kurven durchlaufen werden. Auch die für verschiedene Schichtdicken ermittelten $\beta$-Werte fallen zusammen, wenn sie gegen den Druck aufgetragen werden. Es kann daher angegeben werden:

$$
\beta=0,1097+44,59 N .
$$

$(N=$ Zahl der Mole Jod pro Liter.) Durch Einsetzen dieser $\beta$-Werte in die in I durchgeführten Rechnungen können die Jodmessungen gedeutet werden. Die so berechneten Extinktionskoeffizienten sind in Abb. 1 durch Kreuze markiert worden. Die kleinen Abweichungen zwischen den theoretischen und experimentellen Werten für die Schichtdicken $z=1 \mathrm{~cm}$ und $0,475 \mathrm{~cm}$ sind vermutlich wie die entsprechenden Werte bei den Brommessungen auf Fehler in der Volumen- und Schichtdickenbestimmung der Küvetten zurückzuführen.

Auch die Messungen der $\mathrm{J}_{2}-\mathrm{N}_{2}$-Mischungen sind auf diese Weise auswertbar. Auch hier sind die Linienbreiten linear vom Druck abhängig. Für die $\mathrm{J}_{2}-\mathrm{N}_{2}-$ Mischungen ist Gl. (4) zu erweitern in:

$$
\beta=0,1097+44,59 N+53,15 F .
$$

( $F=$ die Zahl der Mole Stickstoff pro Liter.) Die in -Abb. 3 und 4 eingezeichneten Kreuze wurden mit (5) und I, Abb. 2, berechnet.

Die Berechnung der Gesamtabsorption überlappender Spektrallinien ist also in der Lage, die Messungen mit Joddampf bei $5461 \AA$ darzustellen, obwohl die Voraussetzungen der Herleitung nicht exakt erfüllt sind. Wir möchten noch darauf hinweisen, daß in dieser Auswertung keine weiteren variablen Konstanten benutzt werden. Benutzt wird nur der aus den Experimenten bestimmte Wert von $\bar{\varepsilon}_{\mathrm{H}}=637$. Mit dieser An- gabe allein können die Messungen in so guter Übereinstimmung durch die Theorie erfaßt werden.

Die Deutung der aus (5) ermittelten Linienbreiten mit den in I angegebenen Vorstellungen führt zu physikalisch vernünftigen Ergebnissen. Der druckunabhängige Teil von $\beta\left(\beta_{0}=0,1097\right)$ kann als DopplerBreite gedeutet werden. Entnehmen wir den Angaben Agarbiceanus für das arithmetische Mittel der Linienabstände $d=0,271 \mathrm{~cm}^{-1}$, so folgt daraus und aus der berechneten Doppler-Breite $\beta_{0}=0,1048$. Dieser berechnete Wert für $\beta_{0}$ entspricht einer Gaußschen Linienform, während der ermittelte Wert von 0,1097 einer Dispersionsverteilung der Linienform entspricht. Für die folgenden Berechnungen wollen wir nun annehmen, daß dem aus der Doppler-Breite berechneten Wert von $\beta_{0}=0,1048$ bei den Rechnungen mit Dispersionsform ein $\beta_{0}=0,1097$ zugeordnet werden kann, mit anderen Worten, daß die Benutzung des arithmetischen Mittelwertes für den Abstand $d$ statthaft ist. Würde eine theoretische Berechnung der Gesamtabsorption überlappender Linien bei Überlagerung von Doppler- und Dispersionsform der Linien vorliegen, so könnten aus dem mit unserer Methode ermittelten $\beta_{0}$ auch die Linienabstände genau bestimmt werden. Wegen dieser Überlagerung der beiden Linienformen ist nicht zu erwarten, daß mit Gl. (5) und Gl. I (18) auch noch Jodmessungen mit noch gröBeren Schichtdicken von etwa $300 \mathrm{~cm}$ mit der in Abb. 1 gewonnenen Genauigkeit dargestellt werden können.

Mit dem arithmetischen Mittelwert für $d$ können wir die mittlere Oszillatorenstärke einer Jodlinie zu $f=7,45 \cdot 10^{-7}$ angeben. Die in Abb. 1 dargestellten Messungen zeigen, wie fehlerhaft die Bestimmung der Oszillatorenstärke sein kann, wenn in einem Linienspektrum nicht mit hohen Drucken nach Regel 1 (I) gemessen wird.

Den druckabhängigen Teil der Dämpfungskonstanten in Gl. (5) wird man in erster Näherung nach der Lorentzschen Stoßtheorie deuten. Aus dem LandoltBörnstein ${ }^{22}$ berechnen wir für $210^{\circ} \mathrm{C}$ folgende gaskinetische Durchmesser:

$$
\sigma_{\mathrm{J}_{2}}\left(483^{\circ} \mathrm{K}\right)=6,56^{\circ} \AA, \quad \sigma_{\mathrm{N}_{2}}\left(483^{\circ} \mathrm{K}\right)=3,55 \AA .
$$

Das Verhältnis der gaskinetischen Durchmesser $\frac{\sigma_{\mathrm{J}_{2}-\mathrm{J}_{2}}}{\sigma_{\mathrm{J}_{2}-\mathrm{N}_{2}}}$ hat den Betrag 1,3, wenn für $\sigma_{\mathrm{J}_{2}-\mathrm{N}_{2}}$ das arithmetische Mittel der beiden Einzeldurchmesser genommen wird. Das Verhältnis der optischen Wirkungsdurchmesser

${ }^{22}$ Landolt-Börnstein, Physik.-chem. Tab., 6. Aufl., Bd. I, 1, Springer 1950. 
bei $\mathrm{J}_{2}-\mathrm{J}_{2}$-Begegnungen zum Durchmesser bei $\mathrm{J}_{2}-\mathrm{N}_{2}$ Begegnungen kann mit der Genauigkeit der Gl. (5) zu 1,37 angegeben werden. Diese Angabe ist unabhängig von der Annahme über den mittleren Linienabstand und besitzt die Genauigkeit der Auswertung der Abb. 1 und der Abb. 3. Von einer Resonanz zwischen Eigenmolekülen ist hier also nichts zu beobachten. Das stimmt überein mit den Überlegungen M a r g ena us ${ }^{23}$, nach denen die Resonanzwechselwirkung der Oszillatorenstärke proportional ist, die hier in Molekülspektren klein ist.

Der Absolutwert der optischen Wirkungsdurchmesser kann nicht mit der gleichen Genauigkeit wie die Relativwerte zwischen verschiedenen Gasen angegeben werden. Wir müssen hier wieder die Annahme über den mittleren Linienabstand benutzen. So erhalten wir für die Jodstoßpaare einen optischen Durchmesser von 11,6 $\AA$, also einen um einen Faktor 1,77 größeren Wert als nach der Gaskinetik. Für JodStickstoff-Begegnungen erhalten wir $8,48 \AA$ für die optischen Durchmesser, also um einen Faktor 1,67 höhere Werte als die gaskinetischen Durchmesser.

Es ist vielleicht noch beachtenswert, daß wir mit diesen Messungen und Berechnungen kleinere Wirkungsdurchmesser erhalten, als sie sonst teilweise angegeben werden. $\mathrm{Kond} \mathrm{ratj} \mathrm{ew} \mathrm{und} \mathrm{Polak}{ }^{9}$ geben nach dem schon besprochenen Verfahren für $\sigma_{\mathrm{J}_{2}-\mathrm{N}_{2}}$ $49 \AA$ an. Für $\sigma_{\mathrm{Br}_{2}-\mathrm{N}_{2}}$ berechnen $\mathrm{K}$ ondrat jew und A vra men k o ${ }^{11} 53 \AA$. Elias chewits ch ${ }^{24}$ kommt bei der Berechnung der Energieübertragung bei der Joddampffluoreszenz ebenfalls zu höheren Durchmessern als wir. $\left(\sigma_{\mathrm{J}_{2}-\mathrm{N}_{2}}=52 \AA, \sigma_{\mathrm{J}_{2}-\mathrm{H}_{2}}=20 \AA\right.$. $)$

Elias chewits ch benutzt für seine Berechnungen die Theorie von $\mathrm{S}$ tern und Vollmer ${ }^{25}$. Diese setzt aber ausdrücklich die Gültigkeit des B.G. voraus, das hier nicht gilt. In diesem Zusammenhang ist es bemerkenswert, daß $\mathrm{M}$ a the s o ${ }^{26}$ bei seiner sorgfältigen Untersuchung der CO-Bande im UR auch nur den kleinen Korrektionsfaktor 1,2 bis 1,3 zwischen optischen und gaskinetischen Durchmessern findet. Auch er untersucht die Abweichungen vom L.G. eingehend.

Opechowski ${ }^{21}$ beobachtete bei seinen Feinstrukturuntersuchungen, daß die Gesamtabsorption der Jodlinien stärker als nach dem von $\mathrm{L}$ a d e n b u r g und Reiche angegebenen Wurzelgesetz mit dem Druck zunimmt (vgl. I.). Er vermutete daher, daß die Linienbreite nicht nur nach Lorentz mit dem Druck linear zunimmt, sondern daß sie wegen indu-

23 H. M a r g e n a u, Physic. Rev. 49, 596 [1936].

24 M. E li a s chewits ch, Physik. Z. Sowjetunion 16, 510 [1932]. zierter Prädissoziation noch stärker mit dem Druck zunehmen würde. Wir haben aber zeigen können, daß diese Zunahme der Gesamtabsorption durch die Überlagerungseffekte der Linien zu deuten ist. Wie schon Lochte-Holtgreven ${ }^{27}$ bemerkte, sollte auch die Änderung der Lebensdauer durch Prädissoziation hinsichtlich der Linienbreite nur wie eine zusätzliche Dämpfung wirken. Die Prädissoziation wäre also auch durch die von uns benutzten Formeln zu erfassen. Die Theorie der induzierten Prädissoziation wird häufig bei der Deutung der Halogenspektren herangezogen. Leider werden meist nur qualitative Angaben hierüber gemacht, bei quantitativen Untersuchungen müßten nach der Theorie der Prädissoziation die optischen Wirkungsdurchmesser dann wellenlängenabhängig werden, worüber uns keine genauen Messungen bekannt sind.

Wir haben also mit den hier durchgeführten Überlegungen einige Aussagen über die Feinstruktur der Jodlinien machen können. Diese Aussagen sind nicht als Notbehelf für fehlende Feinstrukturuntersuchungen anzusehen. Die Fehlergrenze bei Feinstrukturuntersuchungen ist für quantitative Aussagen bei stark überlappenden Linien stark erhöht. Zum Beispiel findet A g a r b i c e a n u ${ }^{20}$ bei den Feinstrukturuntersuchungen mit Joddampf keine Proportionalität der Linienbreiten mit dem Fremdgasdruck. Das liegt daran, daß er bei seinen Auswertungen die Überlappung der Linien nicht ohne weiteres berechnen kann. Bei kleinen Drucken, bei denen die Úberlappungen nicht so stark sind, findet er Halbwertsbreiten von $0,03 \mathrm{~cm}^{-1}$ und $0,025 \mathrm{~cm}^{-1}$, für diesen Fall würde aus unseren Berechnungen eine Halbwertsbreite von $0,0291 \mathrm{~cm}^{-1}$ folgen, was also noch innerhalb der Fehlergrenze der Agarbiceanuschen Methode eine Übereinstimmung bedeutet. Am brauchbarsten wäre eine Kombination der Feinstrukturuntersuchung mit der hier beschriebenen Methode der Gesamtabsorption, um exakte Aussagen machen zu können.

An die in I erwähnten Theorien der Linienverbreiterungen ist noch der hier und in II beobachtete Übergang ins Kontinuum anzuschließen. Dieser Übergang erfolgt bei den beiden untersuchten Halogenen, ohne daß eine quadratische Druckabhängigkeit nach der statistischen Theorie (vgl. I) beobachtet werden konnte. Bei großen Drucken war aber nicht nur unsere

25 O. Stern u. M. Voll mer, Physik. Z. 20, 187 [1919].

26 L. A. M a t h e s o n, Physic. Rev. 40, 813 [1932].

27 W. Lochte-Holtgreven, Z. Physik 50, 362 [1936]. 
experimentelle Methodik mit größeren Fehlern behaftet, sondern die genaue Bestimmung der $\beta$-Werte wird hier dadurch erschwert, daß bei hohen Linienbreiten die $\mathrm{zu}$ verschiedenen $\beta$-Werten gehörenden Kurven des Rechenblattes I, Abb. 2, immer enger zusammenlaufen, bis für $\beta>2$ die Bestimmung prinzipiell unmöglich wird. Auch diese Untersuchungen an Joddampf sind eine Stütze dafür, daß die relativ starke Änderung des Spektrums von der Rotationsstruktur zum Kontinuum ohne Änderungen der Übergangswahrscheinlichkeit erfolgt.

Die gute Übereinstimmung zwischen Theorie und Experiment zeigt, daß lediglich durch überlappende Linien ohne sonstige physikalische Besonderheiten die verschiedensten Druckfunktionen der Extinktion vorkommen können. In Abb. 1 steigt z. B. im rechten Teil der Kurve für $z=1 \mathrm{~cm} \bar{\varepsilon}$ ungefähr linear mit dem Druck an. Die Extinktion ist also in diesem Bereich etwa proportional dem Quadrat des Druckes, hier kann also daraus nicht ohne weiteres auf das Vorhandensein von Assoziaten geschlossen werden. Unsere Überlegungen können daher wahrscheinlich auch in anderen Fällen bisher anormal erscheinender Absorptionsbeobachtungen von Nutzen sein.

Herrn Prof. G. Kortü m danke ich für die Anregung zu dieser Arbeit und für Gelegenheit zu Diskussionen, der Notgemeinschaft der Deutschen Wissen$\mathrm{s} c \mathrm{~h}$ a f $\mathrm{t}$ für die Bereitstellung einer Mikrowaage.

\section{Interferenzpunkt-Streuung auf Röntgen-Rückstrahlaufnahmen}

\section{Von Gerhard Frohnmeyer}

Aus dem Röntgeninstitut der Technischen Hochschule Stuttgart und dem Institut für Metallphysik am Max-Planck-Institut für Metallforschung, Stuttgart

(Z. Naturforschg. 6 a, 319_331 [1951]; eingegangen am 11. Mai 1951)

1. Zur Aufklärung der Ursachen der Interferenzpunktstreuung wird eine Versuchsanordnung entwickelt, mit deren Hilfe eine streng monochromatische Röntgenstrahlung erzeugt werden kann, die nur noch $\mathrm{Cu}-\mathrm{K}_{\alpha 1}$ enthält. Dies geschieht

a) durch Monochromatisierung und Fokussierung der Primärstrahlung mit Hilfe eines gebogenen und angeschliffenen Quarzkristalles und durch Ausblendung von $\mathrm{Cu}-\mathrm{K}_{a_{1}}$ genau an der Fokussierungsstelle mittels eines $0,015 \mathrm{~mm}$ breiten Silberspaltes;

b) durch Anwendung eines Doppel-Kristallmonochromators in der Anordnung nach G u in i er.

Die erste Methode erweist sich im vorliegenden Fall trotz größerer Justierungsschwierigkeiten als geeigneter, vor allem weil kürzere Expositionszeiten benötigt werden (12-15 Stdn.).

2. a) Auf Röntgenrückstrahlaufnahmen nach den obigen Verfahren an grobkörnigem, spannungsfreiem Aluminium vom Reinheitsgrad $99,999 \%$ mit reiner $\mathrm{Cu}-\mathrm{K}_{a 1}$-Strahlung wird festgestellt, daß alle Interferenzpunkte auf den zugehörigen Debye-Scherrer-Ring zu liegen kommen. Dies bedeutet, daß alle Kristallite eine einheitliche Gitterkonstante haben innerhalb einer Schwankungsbreite von $\pm 0,05 \mathrm{XE}$.

b) Bei Aufnahmen unter denselben Bedingungen an elektrolytisch hergestelltem und im Vakuum spannungsfrei geglühtem Chrompulver treten Streuungen der einzelnen Interferenzpunkte auf. Dies bedeutet, daß die Gitterkonstanten in den einzelnen Kristalliten selbst und von Kristallit zu Kristallit verschiedene Werte haben. Die Werte der Gitterkonstanten erstrecken sich über ein Intervall von $\pm 0,4 \mathrm{XE}$.

c) Wird das gleiche Chrompulver im Hochvakuum einige Zeit bei einer Temperatur knapp unter seinem Schmelzpunkt (über $2000^{\circ} \mathrm{C}$ ) ausgeglüht und auf diese Weise von eingeschlossenen Gasen befreit, so wird ein einheitlicher Wert der Gitterkonstanten innerha!l' einer Schwankungsbreite von $\pm 0,06 \mathrm{XE}$ erhalten.

3. Die Interferenzpunktstreuung ist also bei sehr reinen Elementen nicht eine Eigenschaft der Stoffe, sondern eine Folge nicht ausreichender Monochromatisierung der Primärstrahlung.

4. Ist die durch die natürliche Linienbreite der Spektrallinien und geometrische Faktoren des Aufnahmeverfahrens bedingte Breite des Debye-Scherrer-Ringes größer als die Ausdehnung der Einzelreflexe, dann liegen auch bei bester Monochromatisierung der Strahlung die einzelnen Reflexe über diesen Bereich verstreut. Zur Ausschöpfung des ganzen Reflexionswinkelbereiches wird die Probe um kleine Winkelbeträge während der Aufnahme gedreht (Kippverfahren).

5. Diese Erkenntnis ermöglicht ein Verfahren, welches Präzisionsmessungen von Gitterkonstanten an grobkristallinen Stoffen auch ohne streng monochromatische Strahlung gestattet. Die Probe wird um kleine Winkelbereiche $\left( \pm 2^{\circ}\right)$ während der Aufnahme gekippt. Dabei ent- 